\title{
Ammonia cores in high mass star formation regions $\star$
}

\author{
Y. Wu ${ }^{1}$, Q. Zhang ${ }^{2}$, W. Yu ${ }^{1,3}$, M. Miller ${ }^{4}$, R. Mao ${ }^{5}$, K. Sun ${ }^{1,4}$, and Y. Wang ${ }^{1,2}$ \\ 1 Astronomy Department, CAS-PKU Joint Beijing Astrophysics Center, Peking Univ., Beijing 100871, PR China \\ e-mail: yfwu@bac.pku.edu.cn \\ 2 Harvard-Smithsonian Center for Astrophysics, 60 Garden St., Cambridge, MA 02138, USA \\ 3 Institute of Inorganische Chemistry, Uni. Bonn, Roemer St., 53117 Bonn, Germany \\ ${ }^{4}$ I, Physics Institut, Köln Univ., Zülpicher St., 50937 Köln, Germany \\ 5 Purple Mountain Observatory, Chinese Academy of Sciences, Nanjing 210008, PR China
}

Received 6 April 2005 / Accepted 3 January 2006

\section{ABSTRACT}

We observed a sample of 35 water masers not coincident with known HII regions and/or low mass young stellar objects (YSOs) with the Effelsberg $100 \mathrm{~m}$ telescope in the $\mathrm{NH}_{3}(J, K)=(1,1),(2,2),(3,3)$ and $(4,4)$ transitions. Sixteen sources were detected in the $\mathrm{NH}_{3}$ emission. The detection rate is $46 \%$. All these sixteen sources have $\mathrm{NH}_{3}(1,1)$ and $(2,2)$ emission, among which four sources have $\mathrm{NH}_{3}(3,3)$ emission. Comparing with the IRAS and the 2MASS data, we analyzed the relationship between the detection rate and the infrared color, the dust temperature and the source distance.

All the detected sources were mapped and 17 cores were obtained (one source IRAS 20215+3725 has two cores). From the detected sources five cores do not coincide with radio continuum or IRAS and MSX point sources. Excluding one core that has no MSX data available, the remaining eleven cores are coincident with IRAS or MSX point sources.

The typical size and mass of the cores are $1.6 \mathrm{pc}$ and $1.5 \times 10^{3} M_{\odot}$, respectively. The average line widths of the $\mathrm{NH}_{3}(1,1)$ and $(2,2)$ are 1.54 and $1.73 \mathrm{~km} \mathrm{~s}^{-1}$. The average kinetic temperature of the gas is about $19 \mathrm{~K}$. These values are much larger than those of low mass cores.

The $\mathrm{NH}_{3}$ cores that coincide with IRAS sources (referred to as Group I) have slightly larger line widths $\left(1.65\right.$ and $1.75 \mathrm{~km} \mathrm{~s}^{-1}$ for the $(1,1)$ and $(2,2)$ lines, respectively) and larger masses $\left(1.8 \times 10^{3} M_{\odot}\right)$ than the mean values of the sample. For this type of core the kinetic temperature correlates with the line width. The line width appears to correlate with the bolometric luminosity and the core size. Despite the average luminosity of $2.9 \times 10^{4} L_{\odot}$, there is no detectable $6 \mathrm{~cm}$ emission. These are candidates for high mass protostars or precursors of UC HII regions. The $\mathrm{NH}_{3}$ cores with peaks offset from infrared sources (referred to as Group II) have an average size of 1.7 pc and an average line width of $1.50 \mathrm{~km} \mathrm{~s}^{-1}$ for the $(1,1)$ line. The line width of the $(1,1)$ emission is smaller than that of the group I. The average mass is $9.4 \times 10^{2} M_{\odot}$. One possible explanation for the deviation is that the $\mathrm{NH}_{3}$ peak and the infrared source correspond to different clumps. These cores are potential high mass star formation sites and may be at an earlier evolutionary stage than those with IRAS point sources. This type of core is seen in mapping observations, and can be easily missed by single-spectrum observations toward the IRAS position.

Key words. masers - ISM: clouds - ISM: kinematics and dynamics - stars: formation

\section{Introduction}

Studies of high mass star formation have drawn more attention in recent years. In particular, the investigation of the initial conditions in massive cores and search for protostellar objects have become active topics in astrophysics. The lack of progress in the past can partly be attributed to the difficulties in identifying protostellar cores. Because of their rapid evolution process, high-mass stars have a short life in the proto stellar phase. Their relatively small population, large distances and complex forming regions make it difficult to "catch" massive stars at early evolutionary stages. Many of current studies

$\star$ Figure 1, Tables 3 and 4 are only available in electronic form at http://www. edpsciences.org of massive star formation regions focus on Ultra Compact HII (UC HII) regions (Churchwell 1999). However, the onset of HII regions may significantly alter the natal molecular cloud, leaving little trace of characteristic conditions prior to or during star formation.

Recently, Molinari et al. (2000, 1998, 1996) investigated 163 IRAS point sources with IRAS colors similar to the compact molecular clouds and $f_{60 \mu \mathrm{m}}>100 \mathrm{Jy}$, but without radio continuum counterparts. $\mathrm{NH}_{3}$ lines, $\mathrm{mm}$ and sub-mm continuum data were obtained. The "Low" sources in the sample, selected with IRAS colors typical of very young stellar objects (YSOs), have associated mm continuum peaks, relatively narrow $\mathrm{NH}_{3}$ line widths, smaller power law indices of the frequency dependence of dust opacity, and a high molecular 
outflow detection rate (Molinari et al. 2000; Zhang et al. 2001). They may be at an evolutionary stage earlier than the UC HII region phase.

Sridharan et al. (2002) and Beuther et al. (2002) examined another group of 69 IRAS point sources. Their sample, associated with the CS $J=2-1$ emission, satisfies the color criteria of UC HII regions. The sources are bright with flux density $f_{100 \mu \mathrm{m}}>500 \mathrm{Jy}$ and $f_{60 \mu \mathrm{m}}>90 \mathrm{Jy}$, but without radio continuum emission at $5 \mathrm{GHz}$. Spectral lines $\mathrm{CO}, \mathrm{NH}_{3}$, and $\mathrm{H}_{2} \mathrm{O}$ and $\mathrm{CH}_{3} \mathrm{OH}$ maser emission revealed that most of the sources have characteristics of evolutionary stage prior to the formation of UC HII regions.

Despite these developments, the number of massive young stars known at very early evolutionary stage is still small. Interstellar water masers are a signpost of the early phases of star formation. In particular, they are frequently found in high-mass star forming regions. The water maser emission may provide another tool to search for early massive cores at early evolutionary stage. With these goals, we started a project that includes an $\mathrm{NH}_{3}$ survey, high density probe $\mathrm{HCN}$ observations, and $\mathrm{mm}$ and $\mathrm{cm}$ continuum emission observations. In this paper, we report our results of the $\mathrm{NH}_{3}$ observations. Sample selection is given in the next section. In Sect. 3 we describe our observations. Sections 4 and 5 present the results and discussions. A summary is given in Sect. 6.

\section{Sample}

Our sources were selected from a complete sample of interstellar water masers in the northern hemisphere (Comoretto et al. 1990; Brand et al. 1994; Valdettaro et al. 2001, and the references therein) using the following criteria:

a. Not coincident with known HII regions from existing surveys by Griffith et al. (1994), Gregory \& Condon (1991), and Altenhoff et al. (1978);

b. IRAS sources in the field not coincident with known lowmass young stellar objects (Wilking et al. 1994; Myers et al. 1983; Terebey et al. 1993; Claussen et al. 1996);

c. IRAS flux density $f_{60} \mu \mathrm{m}>50 \mathrm{Jy}$;

d. No existing $\mathrm{NH}_{3}$ map available in the literature;

e. No overlap with sources in the sample of Molinari et al. (1996);

f. $\operatorname{Dec}>-25^{\circ}$.

Applying these criteria, we selected 35 sources, which are listed in Table 1. The distance is derived from the LSR velocity of the $\mathrm{NH}_{3}(1,1)$ for sources detected in $\mathrm{NH}_{3}$ (see Sect. 4) using the Galaxy rotation curve (Wouterloot \& Brand 1989). For sources not detected in $\mathrm{NH}_{3}$, the distance is quoted from the literature or calculated with the LSR velocity of CO or CS (see notes under Table 1). For objects with distance ambiguity, we exclude the far distance if they lie beyond the scale height $(150 \mathrm{pc})$ of the galactic molecular disk for sources in the Ist galactic quadrant (Molinari et al. 1996, and references therein). If sources within a scale height of $150 \mathrm{pc}$ still have a distance ambiguity, the near distance was adopted. For one source with galactic longitude $l=181^{\circ}, 100 \mathrm{pc}$ was assumed as its distance (Wouterloot \& Brand 1989). There are four sources without any detectable line emission reported in the literature; their distances are not given.

After the $\mathrm{NH}_{3}$ observations we found an additional five sources to be associated with HII regions using other references (Griffith et al. 1991, 1995; Wood \& Churchwell 1989a) or SIMBAD. Three of them were detected (G23.46-0.20, IRAS $18355-0650$ and 20160+3911) in $\mathrm{NH}_{3}$ and two were not (IRAS 05327-0457 and 20144+3526). These results are included in the tables as the last three sources in the detected source list and the last two maps (IRAS 18355-0650 and $20160+3911$ ) of Fig. 1. However they are excluded from the analysis.

\section{Observations}

The observations of the $\mathrm{NH}_{3}(J, K)=(1,1),(2,2),(3,3)$ and $(4,4)$ transitions were made with the Effelsberg $100 \mathrm{~m}$ telescope of the MPIfR during September 11-18, 2002. The beam size is $40^{\prime \prime}$ at this wavelength. The front-end is a dual channel $K$-band HEMT. The system temperatures were $\sim 40 \mathrm{~K}$ to $65 \mathrm{~K}$ during the observations. An autocorrelator with 2048 channels and a total bandwidth of $80 \mathrm{MHz}$ were used. The $K$-band HEMT receiver allows the $\mathrm{NH}_{3}(1,1),(2,2),(3,3)$ and $(4,4)$ lines to be measured simultaneously. The 8 backends were divided into two groups to sample both right and left polarizations (Henkel et al. 2000). The equivalent velocity resolution is $0.25 \mathrm{~km} \mathrm{~s}^{-1}$. NGC 7027 was used for flux calibration with an assumed flux density of $5.86 \mathrm{Jy}$ (Baars et al. 1977), equivalent to a main beam brightness temperature of $7.97 \mathrm{~K}$. The pointing was checked for each target source with nearby continuum sources. The pointing errors were within $5^{\prime \prime}-10^{\prime \prime}$. The position switching mode was used in the observations.

For each source, we started with a five-point mapping in a cross pattern with a grid separation of $40^{\prime \prime}$. If $\mathrm{NH}_{3}$ emission was detected, a more complete map was followed. The integration time was $2.3 \mathrm{~min}$ for each pointing, resulting in a $1 \sigma \mathrm{rms}$ noise of about $0.05 \mathrm{~K}$. The integration time for some spectra was longer because of the overlap of pointings in the mapping.

Data were reduced with the software packages CLASS and GREG.

\section{Results}

Of the 35 sources in the sample, 16 were detected in $\mathrm{NH}_{3}$ emission and were mapped. All the detected sources have $\mathrm{NH}_{3}(1,1)$ and $(2,2)$ line emission. Four (IRAS 18196-1331, IRAS 18352-0148, IRAS 18414-0339, and IRAS 19474+2637) have $\mathrm{NH}_{3}(3,3)$ line emission and 2 (IRAS 18308-0503 and $19363+2018$ ) have possible $(3,3)$ emission. One (IRAS 19474+2637) has possible $(4,4)$ line emission.

The spectra of the $\mathrm{NH}_{3}(1,1)$ and $(2,2)$ lines are fitted to derive the peak intensity, the full width at half maximum, and the optical depth. The fitting takes into account the effect of line broadening from hyperfine splitting. For $\mathrm{NH}_{3}(1,1)$ and $(2,2)$ line broadening effect of the optical depth was also 
Table 1. Observed sources. Columns 2 and 3 give the equatorial coordinates. Columns 4-5 present the galactic coordinates. The $V_{\mathrm{LSR}}$ of the water maser peak velocity is listed in Col. 6. $\mathrm{NH}_{3}$ detection is shown in Col. 7. The last column gives the distance.

\begin{tabular}{|c|c|c|c|c|c|c|c|}
\hline Source Name & RA(1950) & $\operatorname{Dec}(1950)$ & $l\left(^{\circ \prime}\right)$ & $b\left(^{\circ \prime}\right)$ & $V_{p}\left(\mathrm{~km} \mathrm{~s}^{-1}\right)$ & $\mathrm{NH}_{3}$ & $D(\mathrm{kpc})$ \\
\hline RAS $01133+6434$ & 011319.0 & +643454 & 12536 & 206 & -53.0 & $\mathrm{n}$ & $5.2^{1}$ \\
\hline IRAS $02485+6902$ & 024834.5 & +690220 & 13324 & 853 & $-33-15$ & $\mathrm{y}$ & 0.86 \\
\hline IRAS $03035+5819$ & 030333.2 & +581921 & 13955 & 012 & -28.1 & $\mathrm{y}$ & 4.1 \\
\hline IRAS $06001+3014$ & 060010.2 & +301419 & 18050 & 359 & 16.11 & $\mathrm{n}$ & 0.10 \\
\hline IRAS 06306+0437 & 063037.3 & +043716 & 20653 & -156 & 10.08 & $\mathrm{y}$ & 1.7 \\
\hline IRAS $06446+0029$ & 064438.6 & +002926 & 21204 & -044 & 44.89 & $\mathrm{n}$ & $6.1^{3}$ \\
\hline IRAS $06501+0143$ & 065009.8 & +014358 & 21136 & 103 & 55.4 & $\mathrm{y}$ & 6.3 \\
\hline IRAS 07207-1435 & 072043.9 & -143542 & 22934 & 009 & 51.87 & $\mathrm{n}$ & $5.3^{1}$ \\
\hline IRAS 07427-2400 & 074245.0 & -240022 & 24019 & 004 & 70.94 & $\mathrm{n}$ & $6.9^{1}$ \\
\hline IRAS 18061-1927 & 180609.6 & -192756 & 1053 & 008 & 21.7 & $\mathrm{n}$ & $6.1^{4}$ \\
\hline IRAS 18196-1331 & 181937.0 & -133147 & 1738 & 009 & 27.1 & $\mathrm{y}$ & 2.3 \\
\hline IRAS $18265+0028$ & 182632.6 & +002835 & 3050 & 512 & 5.29 & $\mathrm{y}$ & 0.66 \\
\hline IRAS18308-0503 & 183050.8 & -050326 & 2625 & 141 & 53.7 & $\mathrm{y}$ & 3.1 \\
\hline IRAS 18352-0148 & 183514.7 & -014827 & 2949 & 213 & 43.59 & $\mathrm{y}$ & 3.2 \\
\hline IRAS 18361-0627 & 183610.5 & -062744 & 2547 & -008 & 91.1 & $\mathrm{y}$ & 5.7 \\
\hline IRAS 18403-0440 & 184022.0 & -043942 & 2752 & -014 & 9.92 & $\mathrm{n}$ & 3.3 \\
\hline IRAS 18414-0339 & 184128.9 & -033952 & 2853 & -001 & 138.0 & $\mathrm{y}$ & 6.3 \\
\hline IRAS $19303+1651$ & 193016.6 & +165104 & 5239 & -105 & 56.2 & $\mathrm{n}$ & 5.2 \\
\hline IRAS $19363+2018$ & 193620.5 & +201828 & 5622 & -038 & 36.03 & $\mathrm{y}$ & 3.5 \\
\hline IRAS $19471+2641$ & 194706.1 & +264116 & 6307 & 027 & 23.8 & $\mathrm{y}$ & 2.5 \\
\hline IRAS 19474+2637 & 194728.7 & +263737 & 6307 & 020 & 20.53 & $\mathrm{y}$ & 2.5 \\
\hline IRAS $19560+3135$ & 195605.0 & +313557 & 6820 & 119 & -70.9 & $\mathrm{n}$ & \\
\hline IRAS 20110+3321 & 201105.1 & +332111 & 7132 & -024 & 16.35 & $\mathrm{n}$ & \\
\hline IRAS 20144+3726 & 201424.7 & +372622 & 7518 & 119 & -56.7 & $\mathrm{n}$ & \\
\hline IRAS $20215+3725$ & 202135.5 & +372515 & 7606 & 008 & 5.46 & $\mathrm{y}$ & 4.1 \\
\hline IRAS $20306+3749$ & 203041.8 & +374920 & 7729 & -105 & -89.1 & $?$ & $4.4^{5}$ \\
\hline IRAS $21008+4700$ & 210049.1 & +470042 & 8806 & 025 & -49.57 & $\mathrm{n}$ & \\
\hline IRAS $21144+5430$ & 211424.1 & +543057 & 9503 & 358 & -88.21 & $\mathrm{n}$ & $10.3^{1}$ \\
\hline IRAS $21334+5039$ & 213324.0 & +503943 & 9428 & -048 & -43.68 & $\mathrm{n}$ & $5.9^{1}$ \\
\hline IRAS $21379+5106$ & 213755.1 & +510657 & 9518 & -056 & -41.67 & $\mathrm{n}$ & $5.6^{1}$ \\
\hline IRAS $21391+5026$ & 213910.1 & +502612 & 9500 & -135 & -42.6 & $\mathrm{n}$ & $5.6^{1}$ \\
\hline IRAS $21413+5442$ & 214121.2 & +544230 & 9802 & 127 & -59.79 & $\mathrm{n}$ & $7.4^{2}$ \\
\hline IRAS $23004+5642$ & 230027.0 & +564245 & 10829 & -248 & -56.15 & $\mathrm{n}$ & $5.6^{1}$ \\
\hline IRAS 23139+5939 & 231358.3 & +593906 & 11115 & -046 & -59.6 & $\mathrm{y}$ & 4.4 \\
\hline IRAS $23151+5912$ & 231508.6 & +591225 & 11114 & -114 & -53.7 & $\mathrm{y}$ & 5.5 \\
\hline IRAS $05327-0457^{\circ}$ & 053246.4 & -045738 & 20840 & -1911 & 10.17 & $?$ & $1.3^{2}$ \\
\hline $\mathrm{G} 23.46-0.20^{\circ}$ & 183201.1 & -083333 & 2327 & -012 & 47.2 & $\mathrm{y}$ & 6.0 \\
\hline IRAS $18355-0650^{\circ}$ & 183532.6 & -065028 & 2523 & -011 & 65.08 & $\mathrm{y}$ & 5.7 \\
\hline IRAS $20144+3526^{\circ}$ & 201427.5 & +352647 & 7339 & 012 & -56.7 & $\mathrm{n}$ & $11^{5}$ \\
\hline IRAS $20160+3911^{\circ}$ & 201605.2 & +391122 & 7656 & 202 & -5.55 & $\mathrm{y}$ & 3.7 \\
\hline
\end{tabular}

Note: ${ }^{\circ}$ Denotes the sources associated with HII region and not included in the analysis; 1 . Wouterloot \& Brand (1989); 2 . Tatematsu et al. (1993); 3. Wu al. (2001); 4. Scoville et al. (1987); 5. Bronfman et al. (1996).

taken into account (Wouterloot, private communication). The observed parameters are summarized in Table $2 . \mathrm{NH}_{3}$ spectra and the integrated emission of each source are presented in Fig. 1. All the peak intensities are listed in Table 2 as $P(1,1)$, $P(2,2)$ and $P(3,3)$.

Following Harju et al. (1993), we obtained the core size, $T_{\text {ex }}$, total column density $N\left(\mathrm{NH}_{3}\right)$, and rotation temperature. Local molecular hydrogen densities $n\left(\mathrm{H}_{2}\right)$ were calculated using the statistical equilibrium equations given by
Ho \& Townes (1983). The hydrogen densities can be obtained from the $\mathrm{NH}_{3}$ density by assuming the ammonia fractional abundance, which can vary from $10^{-8}$ to $10^{-7}$ according to the study of Orion clumps (Harju et al. 1993). We adopted the value of $\left[\mathrm{NH}_{3}\right] /\left[\mathrm{H}_{2}\right]=3 \times 10^{-8}$. In addition, molecular hydrogen densities $n\left(\mathrm{H}_{2}\right)$ were also calculated using the statistical equilibrium equations given by Ho \& Townes (1983). The kinetic temperatures were solved following Walmsley \& Ungerechts (1983), modified with the new collisional rate coefficients by 


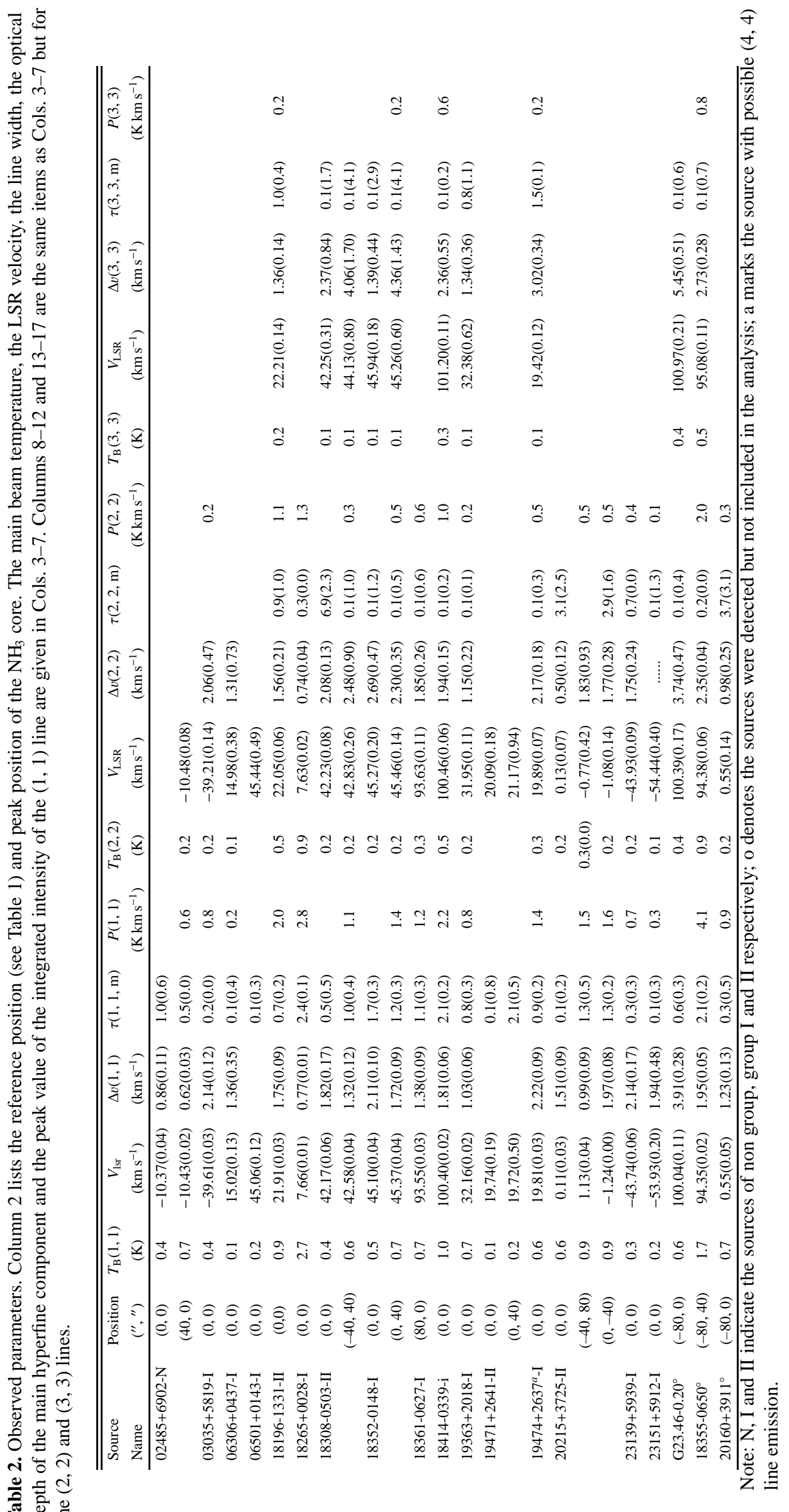



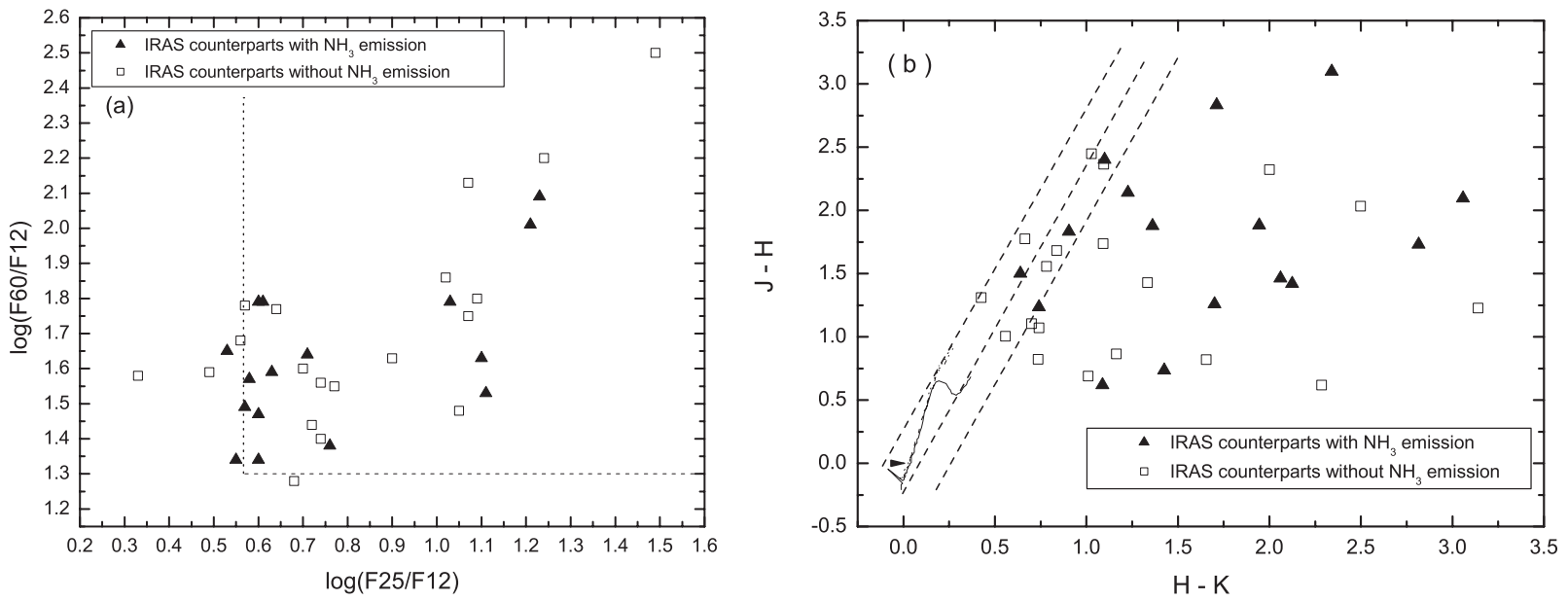

Fig. 2. IRAS color-color diagram a) and $2 \mathrm{MASS}$ color-color diagram b). The solid triangles indicate sources detected in $\mathrm{NH}_{3}$ emission while the open squares denote sources without $\mathrm{NH}_{3}$ emission. The dotted lines in the left panel indicate the color box of UCHII regions (Wood \& Churchwell 1989b). In the right panel, the solid curve shows the infrared color of the main sequence and giant branch (Bessell \& Brett 1988); the dotted line separates the sources with infrared excesses from the reddened main-sequence stars allowing for errors in the colors.

Danby et al. (1988); clump mass was derived following Wiseman \& Ho (1998), and the virial mass was obtained using the relation in Estalella et al. (1993). The derived quantities are listed in Table 3.

\section{Discussion}

\subsection{Detection rate}

Of the 35 sources in the sample, $\mathrm{NH}_{3}$ emission was detected towards 16 sources. The detection rate is $46 \%$. This is lower than those of the "High" sources of Molinari et al. (1996) and the sample in Sridharan et al. (2002). We investigated the relation between the detection rate and the infrared colors of the associated sources. Table 4 lists the parameters of the IRAS sources and the 2MASS counterparts chosen as the reddest and brightest point source at the $K$ band within the $1^{\prime}$ radius of the IRAS source. Figure 2 presents the color-color diagram of both IRAS and 2MASS sources for the sample (data are in Table 4). The distribution of the two types of sources in both diagrams are similar. Overall these maser sources are very red in the infrared colors and most of them satisfy the criteria of UC HII regions (Wood \& Churchwell 1989b).

The infrared color indices do not seem to correlate with the occurrence rate of water maser emission. We also investigated the dust temperature. The average value of the IRAS sources coincident with $\mathrm{NH}_{3}$ cores is $35 \mathrm{~K}$; that of the IRAS sources offset from the $\mathrm{NH}_{3}$ cores or without detectable $\mathrm{NH}_{3}$ emission is $38 \mathrm{~K}$. The temperature difference is not significant.

The relation between the detection rate and the distance was also investigated. Figure 3 a shows the frequency distribution of detected (triangle) and non-detected sources (open square) with distance. Figure $3 \mathrm{~b}$ presents the detection rate versus the distance. When $D>6 \mathrm{kpc}$ the detection rate is low. This is likely due to the effect of beam dilution (Molinari et al. 1996).

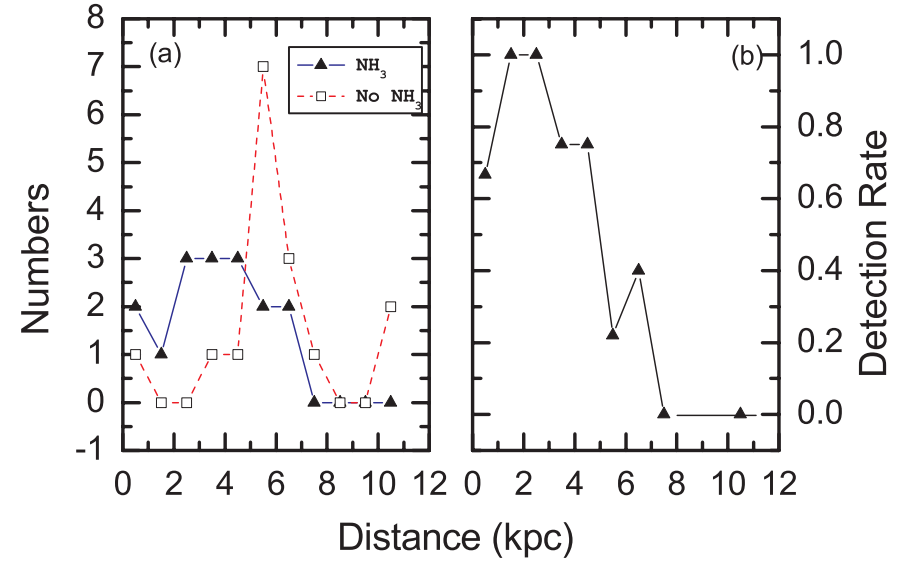

Fig. 3. a) Number of sources detected (solid triangle) and non-detected (open square) in $\mathrm{NH}_{3}$ as a function of source distance. b) The detection rate of $\mathrm{NH}_{3}$ vs. source distance.

\subsection{Core identification-candidate precursors of UC HII regions and high mass cores at even earlier evolutionary stages}

Among the 16 sources mapped in $\mathrm{NH}_{3}, 10$ sources have $\mathrm{NH}_{3}$ cores that peak within the error ellipse of the IRAS position; one of them, IRAS $18265+0028$, has a minor core $80^{\prime \prime}$ west of the IRAS source. For the remaining 6 sources $(02485+6902$, 18196-1331, 18308-0503, 18361-0627, 19471+2641 and $20215+3725$ ), the $\mathrm{NH}_{3}$ core peaks are offset from the IRAS source by at least one beam or outside of the IRAS error ellipses. One of them (IRAS $20215+3725$ ) has two $\mathrm{NH}_{3}$ cores between which the IRAS source is located. The lack of IRAS sources in these cores suggests that $\mathrm{NH}_{3}$ clumps in these maser sources may be at a different evolutionary phase.

To further examine the $\mathrm{NH}_{3}$ cores without IRAS sources, we investigate their association with stellar sources using the mid infrared database from the Midcourse Space Experiment (MSX) (Egan et al. 1998; Carey et al. 1998). We made maps 

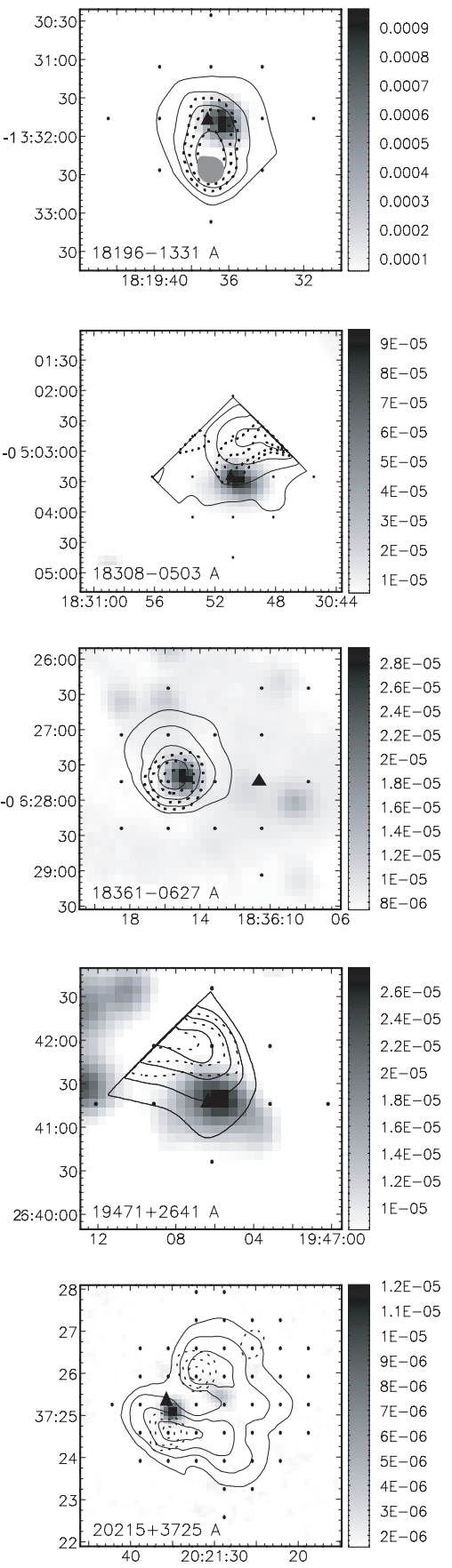
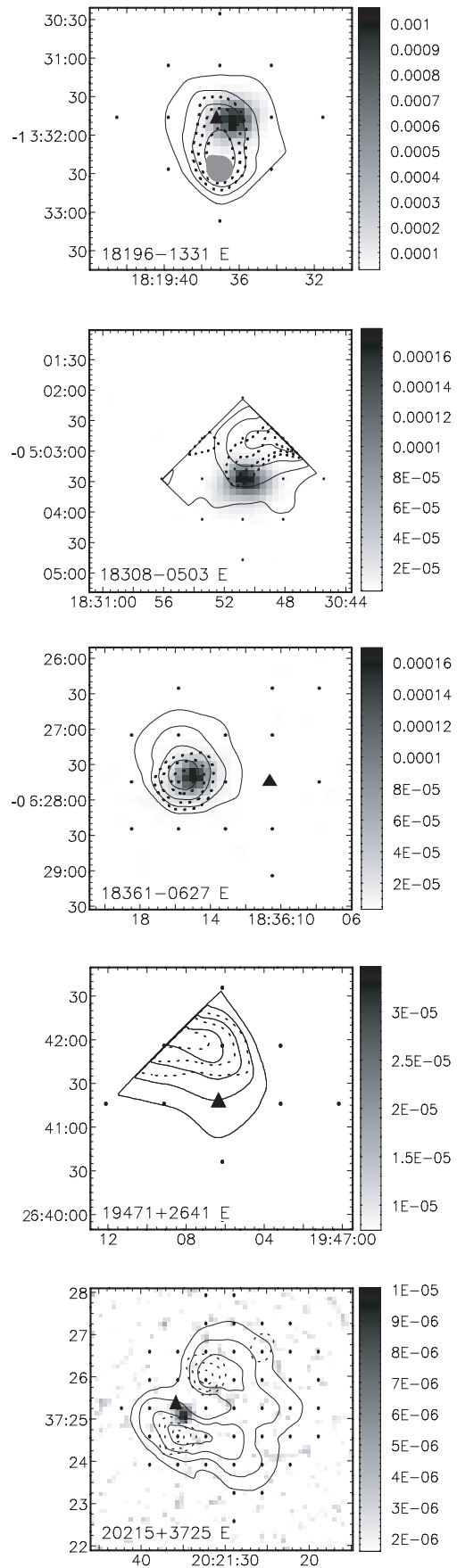

Fig. 4. Maps of MSX and $\mathrm{NH}_{3}$ for cores offset from the IRAS position. The triangle marks the position of the IRAS source. Left: the overlay of $\mathrm{NH}_{3}$ contours on the MSX image in the band A (grey scales); Right: same as the left panel but for the MSX band E in grey scale. The MSX flux is in $\mathrm{W} \mathrm{m}^{-2} \mathrm{sr}^{-1}$. The source name is indicated at the lower-left corner of each panel.

covering the $\mathrm{NH}_{3}$ emission region at the $\mathrm{A}(8.28 \mu \mathrm{m})$ and $\mathrm{E}$ $(21.3 \mu \mathrm{m})$ bands of MSX. All six sources have MSX data available except for IRAS 02485+6902. The results are shown in Fig. 4. For one source, IRAS 18361-0627, the $\mathrm{NH}_{3}$ emission peak is coincident with an MSX source while the other five cores do not correspond to any mid infrared source.

Excluding IRAS $02485+6902$ for which no MSX data is available, we can divide the mapped sources into two groups. Group I contains 11 sources for which the $\mathrm{NH}_{3}$ cores peak toward the IRAS or MSX (IRAS 18361-0627) point sources, and water masers. These objects have a high far infrared luminosity of $2.9 \times 10^{4} L_{\odot}$ (see Table 5), but no detectable freefree emission at $6 \mathrm{~cm}$ wavelengths. This group is analogous to high mass protostellar objects or the precursors of UC HII regions (Molinari et al. 1996, 2000; Sridharan et al. 2002; Beuther et al. 2002). Another group (Group II) has 4 sources (5 cores). Unlike the sources in group I whose $\mathrm{NH}_{3}$ core is coincident with the infrared source, the sources in group II do not have far and mid infrared sources associated with the $\mathrm{NH}_{3}$ emission peak according to the IRAS and MSX catalogues. Their physical parameters (Tables 2 and 3, also see next section) show that these cores are massive and dense. They may 
Table 5. Average properties of $\mathrm{NH}_{3}$ cores.

\begin{tabular}{lrrrrrcc}
\hline \hline Name & $d(\mathrm{pc})$ & $T_{k}(\mathrm{~K})$ & $\Delta V(1,1)\left(\mathrm{km} \mathrm{s}^{-1}\right)$ & $\Delta V(2,2)\left(\mathrm{km} \mathrm{s}^{-1}\right)$ & \multicolumn{1}{c}{$M\left(M_{\odot}\right)$} & $D(\mathrm{pc})$ & $L_{\mathrm{bol}}\left(L_{\odot}\right)$ \\
\hline Total & $1.55 \pm 0.80$ & $19.0 \pm 4.6$ & $1.54 \pm 0.50$ & $1.73 \pm 0.45$ & $1500 \pm 1600$ & $3580 \pm 1720$ & $25900 \pm 27300$ \\
Group I & $1.61 \pm 0.76$ & $19.7 \pm 4.7$ & $1.65 \pm 0.47$ & $1.75 \pm 0.50$ & $1800 \pm 1900$ & $4000 \pm 1900$ & $29000 \pm 28000$ \\
Group II & $1.66 \pm 0.72$ & $17.7 \pm 3.9$ & $1.50 \pm 0.38$ & $1.67 \pm 0.11$ & $940 \pm 380$ & $3200 \pm 830$ & $(24000 \pm 25000)^{3}$ \\
${\text { Cepheus (far })^{1}}^{1}$ & $0.96 \pm 0.38$ & $20.4 \pm 3.7$ & $1.5 \pm 0.4$ & & $590 \pm 910$ & 3500 & $25000 \pm 30000$ \\
Taurus $^{2}$ & $0.10 \pm 0.04$ & $10.0 \pm 2.1$ & $0.30 \pm 0.07$ & & $9 \pm 11$ & 140 & $1.1 \pm 1.4$ \\
\hline
\end{tabular}

Note: 1. Harju et al. (1993); 2. Benson \& Myers (1989), data taken from ref. 1 directly; 3. The value in parentheses is the luminosity of the IRAS sources for the group II.
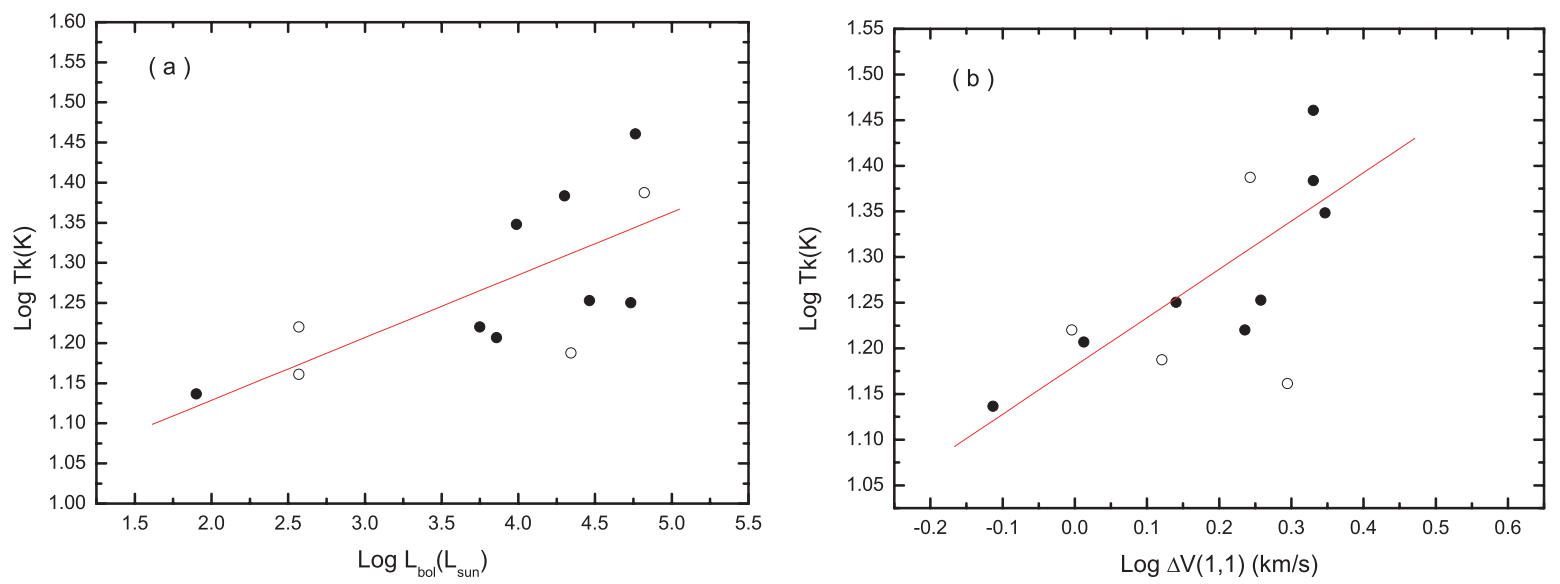

Fig. 5. a). The relation between the kinetic temperature and bolometric luminosity. The filled circle represent sources with $\mathrm{NH}_{3}$ cores peak at the IRAS sources, the open circles indicate sources with the core peaks offset from the IRAS sources. b). Same as a) but for the relation between the kinetic temperature and the $(1,1)$ line width. The line is a least squares fit to the filled circles.

contain new high mass star formation sites which are likely at an earlier evolutionary stage than those found in group I sources (see Sect. 5.3).

\subsection{Physical properties of the two types of cores}

The velocity $\left(V_{\text {lsr }}\right)$ of the $\mathrm{NH}_{3}$ emission is in good agreement with that of the water masers or is within the emission range of the masers (see Tables 1 and 2), suggesting that these two phenomena are associated. However, a comparison of their spatial relation requires high resolution observations.

The sizes of the $(1,1)$ cores range from 0.40 to $3.1 \mathrm{pc}$ with an average of $1.6 \mathrm{pc}$ for group I and $1.7 \mathrm{pc}$ for group II, respectively. Both are much larger than that of the nearby dense cores (0.1 pc on average) (Myers \& Benson 1983). The sizes of the $(2,2)$ emission are smaller than those of the $(1,1)$ emission for all the sources.

The rotation temperature of $(1,1)-(2,2), T_{R 21}$, is calculated for all the sources with $\mathrm{NH}_{3}$ detection. For sources with $(3,3)$ line emission the rotation temperature of $(2,2)-(3,3), T_{R 32}$, is also calculated. Comparing $T_{R 21}$ and $T_{R 32}$ we can see that the core is warmer inside for sources with $(3,3)$ emission. Among the 6 sources with $\mathrm{NH}_{3}(3,3)$ or possible $(3,3)$ emission, four are in group I. In group II, IRAS 18196-1331 has $(3,3)$ emission and $18308-0503$ has possible $(3,3)$ line emission.

The kinetic temperature of the $\mathrm{NH}_{3}$ cores ranges from 14 to $30 \mathrm{~K}$ (better than the $3 \sigma$ level, same as below). The average value is $19 \mathrm{~K}$ (excluding $S / N \leq 3 \sigma$ ). This is slightly lower than that of both the "High" (22.4 K) and "Low" (21.7 K) groups of Molinari et al. (1996). The $T_{k}$ is much higher than the average of $11 \mathrm{~K}$ for low mass sources (Myers \& Benson 1983) but lower than that of the cores in UC HII regions, which is $28 \mathrm{~K}$ obtained from Churchwell et al. (1990). For sources in group I and II the average values are 20 and $18 \mathrm{~K}$, respectively. The difference in the two groups is not obvious.

Figure 5a presents a plot of $T_{k}$ vs. bolometric luminosity. The parameters at the peak position of the cores are used (data are similar to or better than the $3 \sigma$ level, as below). The filled and open circles indicate sources in group I and II, respectively. Since the sample of group II is too small for statistical analysis we present them in the figure to display their distribution. The least squares linear fit is for group I only, which is $\log T_{k}=0.97 \pm 0.14+(0.08 \pm 0.03) \log L_{\text {bol }}$. The correlation coefficient $r$ is 0.67 . It shows that $T_{k}$ is correlated with bolometric luminosity of the associated sources: the internal sources may be a heating mechanism of the gas and dust in cores for group I. The plot of $T_{k}$ Vs. $(1,1)$ line width is shown in Fig. 5b. The solid line is the least squares linear fit for group I: $\log T_{k}=1.18 \pm 0.03+(0.53 \pm 0.14) \log \Delta V(1,1)$ with $r=0.83$. This suggests that turbulence heating may be also effective for these cores (Gusten et al. 1985).

For the 17 detected cores, the widths of the $(1,1)$ and $(2,2)$ lines range from 0.50 to more than $3 \mathrm{~km} \mathrm{~s}^{-1}$, which cannot have been caused by thermal motion in these cores (Molinari et al. 1996, and the references therein) and are clearly different from those of low mass dense cores (Myers \& Benson 1983). The 

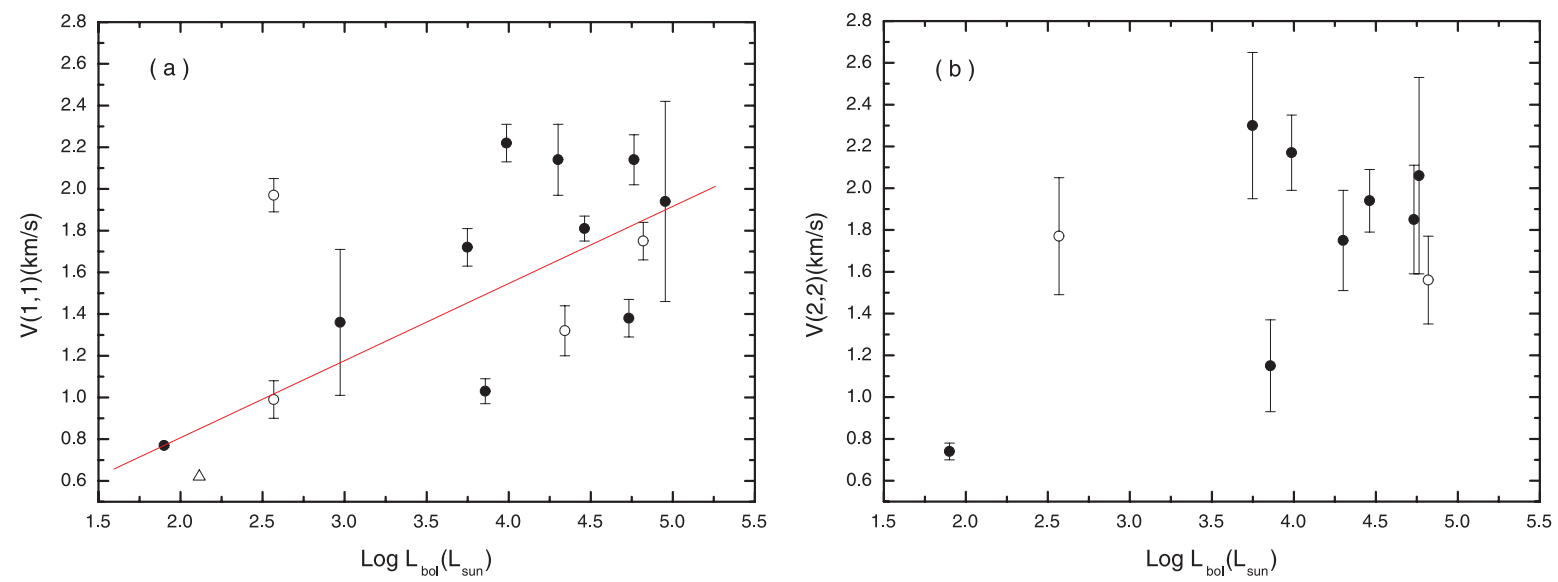

Fig. 6. The line width vs. bolometric luminosity. a) is for the $(1,1)$ line and $\mathbf{b})$ is for $(2,2)$ line. The symbols are the same as in Fig. 5 . The line in a) is a least squares fit for the filled circles.

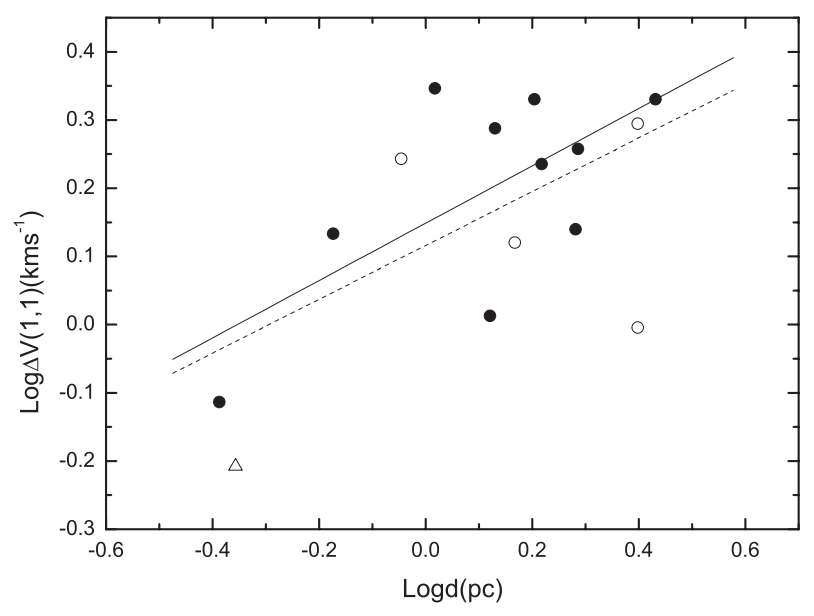

Fig. 7. Line width vs. the source size. The filled and the open circles are the same as in Fig. 5. The open triangle denotes the IRAS $02485+6902$ which was not classified. The solid line is a least squares fit for the filled circles and the dashed line for the whole sample.

average $\Delta V(1,1)$ and $\Delta V(2,2)$ are $1.54 \mathrm{~km} \mathrm{~s}^{-1}$ and $1.73 \mathrm{~km} \mathrm{~s}^{-1}$, respectively.

Figure 6 presents the line width versus bolometric luminosity of the associated IRAS sources. One can see from Figs. 6a and $6 \mathrm{~b}$ that only $\Delta V(1,1)$ and $L_{\text {bol }}$ are correlated. The least squares linear fit for sources in group I gives $\Delta V(1,1)=(0.20 \pm$ $0.56)+(0.37 \pm 0.14) \log L_{\text {bol }}$. In Fig. $6 a$, the distribution of the group II sources shows a similar trend. Such a correlation was also seen in samples in Wouterloot et al. (1988), and may be partly due to the effect of source distance.

Figure 7 plots the $(1,1)$ line width versus the core size. The solid line indicates the least squares linear fit for group I: $\log \Delta V(1,1)=(0.15 \pm 0.04)+(0.42 \pm 0.16) \log d$ with $r=$ 0.66 . If all the sources are included, the relation is shown as the dashed line with $\log \Delta V(1,1)=(0.11 \pm 0.04)+(0.39 \pm$ $0.15) \log d$ and $r=0.59$. The relation is consistent with that of Larson (1981). The results show that the line width and the size of the cores is correlated, suggesting that the turbulence broadening may be important in the group I sources, and also

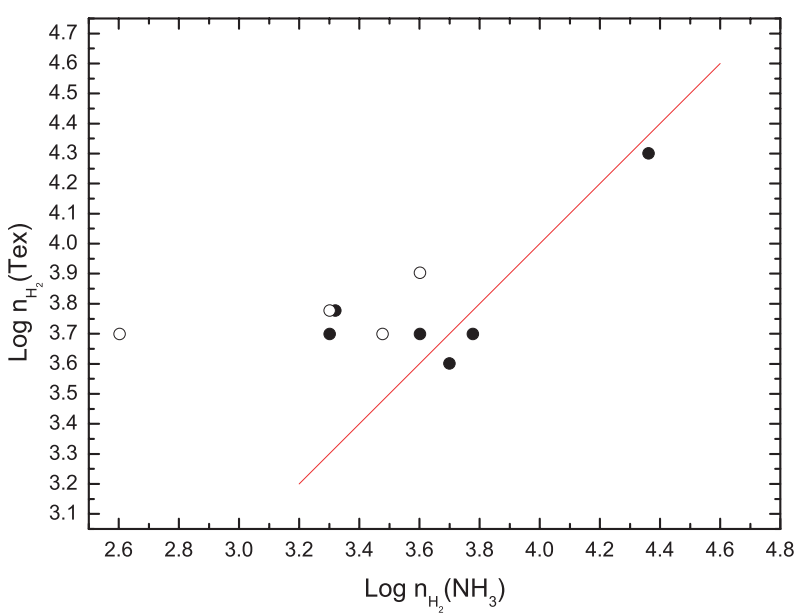

Fig. 8. $\mathrm{H}_{2}$ density calculated from the statistical equilibrium, $n_{\mathrm{H}_{2}}$, vs. that from the ammonia column density, $n_{\mathrm{H}_{2}}\left(\mathrm{NH}_{3}\right)$. The symbols are the same as in Fig. 5. The line indicates where the two densities are the same.

effective in group II sources. Larger samples are needed to better constrain the correlation.

For IRAS 18196-1331 and 18414-0339 the line profiles deviate from the Gaussian shape, showing a narrow peak and broad line wings. The VLA study of IRAS 20126+4104 suggested that such profile can be produced in a disk+core system (Zhang et al. 1998), by superposition of the narrow and strong emission from the core, and the weak and broad emission from the compact disk. This type of line profile is present in both $(1,1)$ and $(2,2)$ lines in IRAS 18196-1331 and 18414-0339 (see Fig. 1). Higher spatial resolution observations are needed to resolve the profiles.

Ten sources have $\mathrm{H}_{2}$ density, $n_{\mathrm{H}_{2}}\left(\mathrm{NH}_{3}\right)$, derived from ammonia column density, and $\mathrm{H}_{2}$ density, $n_{\mathrm{H}_{2}}$, derived from the statistical equation (see Ho \& Townes 1983). Figure 8 shows a comparison of $n_{\mathrm{H}_{2}}$ and $n_{\mathrm{H}_{2}}\left(\mathrm{NH}_{3}\right)$. The average values of $n_{\mathrm{H}_{2}}$ and $n_{\mathrm{H}_{2}}\left(\mathrm{NH}_{3}\right)$ are $6.9 \times 10^{3} \mathrm{~cm}^{-3}$ and $5.1 \times 10^{3} \mathrm{~cm}^{-3}$, respectively. The difference of the two values is less than that of the large sources of Harju et al. (1993). 
The masses of the $\mathrm{NH}_{3}$ cores range from a few hundred to a few thousand $M_{\odot}$ and the average value is $1.5 \times 10^{3} M_{\odot}$. The masses are different from those of low mass cores whose average mass is $4 M_{\odot}$ (Myers \& Benson 1983). For sources with accurate $\Delta V(>3 \sigma)$, virial masses were calculated with the relation (Estalella et al. 1993):

$$
M_{\mathrm{vir}}=210(R / \mathrm{pc})\left(\Delta V / \mathrm{km} \mathrm{s}^{-1}\right)^{2}\left(M_{\odot}\right) .
$$

The average ratio of $M / M_{\mathrm{vir}}$ is 3.7. For the ten sources with available $n_{\mathrm{H}_{2}}\left(\mathrm{NH}_{3}\right)$ and $n_{\mathrm{H}_{2}}$, the $\mathrm{H}_{2}$ density derived from the virial masses $n_{\text {vir }}$ is $1.2 \times 10^{3} \mathrm{~cm}^{-3}$ which is less than both the $n_{\mathrm{H}_{2}}$ and $n_{\mathrm{H}_{2}}\left(\mathrm{NH}_{3}\right)$. There are two sources with $M_{\mathrm{vir}}>M$, suggesting that for these cores the gravity is less than the internal support. The comparison is complicated by the uncertainty of the ammonia abundance adopted. The dynamic status of these sources needs further investigation.

The physical characteristics of the cores are summarized in Table 5. For comparison, the corresponding parameters of sources in Cepheus and Taurus (Harju et al. 1993; Benson \& Myers 1989) are also listed. One can see that the size and mass of our sources are much larger than those of the low mass cores in Taurus (Benson \& Myers 1989), and larger than those of high mass sources in Cepheus (Harju et al. 1993). The sources in the two groups have a similar size on average. However group II sources tend to have narrower line widths, and smaller masses. The physical parameters and their relations show that the group I cores are candidates of high mass protostellar objects (Molinari et al. 1996; Beuther et al. 2002). Cores in group II do not contain IRAS sources and MSX sources and also have large masses $\left(9.4 \times 10^{2} M_{\odot}\right)$.

The observed dispersion of the IRAS point source with respect to the core peak is common in star formation regions (Clark 1987; Harju et al. 1993; Wei et al. 2005). One possible explanation for this deviation is that stars move away from the cores (Harju et al. 1993; Clark 1987). For the sources with deviations between the IRAS positions and the core peaks in this paper, a preliminary analysis shows that the case may be different:

1. From Fig. 4 one can see that the distribution of the IRAS and the MSX sources is different. Toward IRAS 18361-0627, the core peak overlaps with an MSX source although it is deviated from the IRAS source. Toward 19472+2641, there are several MSX sources around the core and it is difficult to determine which ones moved out from the core. These factors introduce an uncertainty in the statistical analysis of the deviation between the infrared source and the core center based on the dispersion of the IRAS position with respect to the core peak.

2. The core peak and the IRAS position may correspond to different gas clumps. The angular offset between the $\mathrm{NH}_{3}$ peak and the IRAS position toward IRAS 18196-1331 is the smallest one among the five $\mathrm{NH}_{3}$ cores. Recent VLA observations of the $\mathrm{NH}_{3}(1,1)$ and $(2,2)$ revealed several $\mathrm{NH}_{3}$ clumps in this region (Wang et al. 2005). The IRAS position is offset from these clumps. It seems that the IRAS source might be more evolved and has dispersed the parental clump, while the present clumps may be massive pre-protostellar cores or pre-proto-cluster cores (Evans et al. 2002).

This scenario shows that the IRAS sources in group II may be more evolved than those in group I since the young stellar sources had time either to move away from the core center or to disperse the surrounding gas. The cores of group II may be at an earlier stage than those containing IRAS and MSX sources. High resolution observations of both molecular lines and infrared emission are required for a more detailed study.

\subsection{Observational method-comparing to surveys of similar samples}

The criteria of our sample are the same as Molinari et al. (1996) and Sridharan et al. (2002) except that the limit of the IRAS flux density is lower. However, the far infrared luminosities in all three samples are similar. Our survey resulted in two kinds of cores: the group I cores are similar to those in Molinari et al. (1996) and Sridharan et al. (2002). These massive cores, containing IRAS sources with an average $L_{\text {bol }}$ of $2.9 \times 10^{4}$ but without $6 \mathrm{~cm}$ emission, are the candidates of precursors of UC $\mathrm{HII}$ regions. The group II cores have $\mathrm{NH}_{3}$ emission peaks deviated from the dominant IRAS sources in the mapping regions. Detection of these cores are largely due to mapping of the region, since the $\mathrm{NH}_{3}$ emission would be easily missed by any single pointing observation centered at the IRAS position.

\section{Summary}

$\mathrm{NH}_{3}(1,1),(2,2),(3,3)$ and $(4,4)$ lines were observed toward 35 water maser sources excluding known HII regions and low mass sources. Sixteen sources were detected in $\mathrm{NH}_{3}$. The detection rate of $\mathrm{NH}_{3}$ is $46 \%$, and has a dependence on the source distance. All the detected sources have $(1,1)$ and $(2,2)$ line emission. Six sources have $(3,3)$ or possible $(3,3)$ lines and one has possible $(4,4)$ lines.

The detected sources were all mapped and 17 cores were obtained (IRAS 20215+ 3725 has two cores). Five cores (group II) (IRAS 18196-1331, 18308-0148, 19471+2641 and 20215NW, 20215SSE) have the $\mathrm{NH}_{3}$ emission peaks offset from the IRAS and MSX point sources. Eleven sources (group I) have IRAS or MSX sources towards the core center.

The average kinetic temperatures of both groups are lower than that of cores in UC HII regions but higher than that of low mass cores. Group I has $T_{k}$ values similar to the "low" group of Molinari et al. (1996). It is possible that they are heated by internal sources and/or turbulence.

The $(1,1)$ and $(2,2)$ line widths of the group II sources are smaller than those of the group I sources, but both are much larger than those of the low mass cores. For the group I sources the $(1,1)$ line widths are correlated with the bolometric luminosity of the associated sources. The $(1,1)$ line widths and the core sizes appear correlated.

The average $\mathrm{H}_{2}$ density derived from the statistical equilibrium (Ho \& Townes 1983) is nearly 1.4 times of that of the $\mathrm{NH}_{3}$ column density. Both are higher than the density of the virial mass. The average sizes for cores of group I and II 
are 1.6 and $1.7 \mathrm{pc}$, respectively. The typical core mass of the entire sample is $1.5 \times 10^{3} M_{\odot}$. For group II sources, the core mass is lower than that of group I.

The general properties of our cores associated with IRAS sources suggest that the group I cores are high mass protostellar candidates. Cores in group II are offset from infrared sources. The IRAS sources in group II might be more evolved than those of group I. Evidence shows that the gas core peak and the infrared source of group II may correspond to different gas clumps. These cores may be younger than the protostellar stages and may be candidates of high mass pre-protostellar cores or pre-proto-cluster cores.

Acknowledgements. We are very grateful to Dr. C. Henkel and the staff at the Effelsberg for the excellent support during the observations. We thank L., Zhu, J. Esembek and J. Wang for the help in figure preparation and source selection. We would like to express our thanks to the anonymous referee for valuable suggestions and detail comments. The paper is based on observations with the 100-m telescope of MPIfR (Max-Planck-Institute for Radioastronomy) at Effelsberg. This research is supported by the Grants 10128306, 10133020, 10203003, 10373025 and NKBRSF G1999075405.

\section{References}

Altenhoff, W. J., Downes, D., Pauls, T., \& Schraml, J. 1978, A\&AS, 35,23

Baars, J. W. M., Genzel, R., Pauliny-Toth, I. I. K., \& Witzel, A. 1977, A\&A, 61, 99

Benson, P. J., \& Myers, P. C. 1989, ApJS, 71, 89

Bessell, M. S., \& Brett, J. M. 1988, PASP, 100, 1134

Beuther, H., Schilke, P., Menten, K. M., et al. 2002, ApJ, 566, 945

Brand, J., Cesaroni, R., Caselli, P., et al. 1994, A\&AS, 103, 541

Bronfman, L., Nyman, L.-A., \& May, J. 1996, A\&AS, 115, 81

Carey, S. J., Clark, F. O., Egan, M. P., et al. 1998, ApJ, 508, 721

Churchwell, E., Walmsley, C. M., \& Cesaroni, R. 1990, A\&AS, 83, 119

Churchwell 1999, in The Origin of Stars and Planetary Systems, ed. C. J. Lada, \& N. D. Kylafis (Kluwer Academic Publishers), 515

Clark, F. O. 1987, A\&A, 180, L1

Claussen, M. J., Wilking, B. A., Benson, P. J., et al. 1996, ApJS, 106, 111

Comoretto, G., Palagi, F., Cesaroni, R., et al. 1990, A\&AS, 84, 179

Danby, G., Flower, D. R., Valiron, P., Schilke, P., \& Walmsley, C. M. 1988, MNRAS, 235, 229

Egan, M. P., Shipman, R. F., Price, S. D., Carey, S. J., \& Clark, F. O. 1998, ApJ, 494, L199
Estalella, R., Mauersberger, R., Torrelles, J. M., et al. 1993, ApJ, 419, 698

Evans, N. J. II, Shirley, Y. L., Mueller, K. E., \& Knez, C. 2002, in The Earliest Stages of Massive Star Birth, ed. P. A. Crowthe (San Francisco, ASP), ASP Conf. Ser., 267, 17

Gregory, P. C., \& Condon, J. J. 1991, ApJS, 75, 1011

Griffith, M. R., Langston, G., Heflin, M., Conner, S., \& Burke, B. 1991, ApJS, 75, 801

Griffith, M. R., Wright, A. E., Burke, B. F., \& Ekers, R. D. 1994, ApJS, 90, 179

Griffith, M. R., Wright, A. E., Burke, B. F., \& Ekers, R. D. 1995, ApJS, 97, 347

Gusten, R., Walmsley, C. M., Ungerechts, H., \& Churchwell, E. 1985, A\&A, 142, 381

Harju, J., Walmsley, C. M., \& Wouterloot, J. G. A. 1993, A\&AS, 98, 51

Henkel, C., Mauersberger, R., Peck, A. B., Falcke, H., \& Hagiwara, Y. 2000, A\&A, 361, 45

Ho, P. T. P., \& Townes, C. H. 1983, A\&AR, 35, 23

Larson, R. B. 1981, MNRAS, 194, 809

Molinari, S., Brand, J., Cesaroni, R., \& Palla, F. 1996, A\&A, 308, 573

Molinari, S., Brand, J., Cesaroni, R., Palla, F., \& Palumbo, G. G. C. 1998, A\&A, 336, 339

Molinari, S., Brand, J., Cesaroni, R., \& Palla, F. 2000, A\&A, 355, 617

Myers, P. C., \& Benson, P. J. 1983, ApJ, 266, 309

Myers, P. C., Linke, R. A., \& Benson, P. J. 1983, ApJ, 264, 517

Scoville, N. Z., Yun, M., Sanders, D. B., Clemens, D. P., \& Waller, W. H. ApJS, 63, 821

Sridharan, T. K., Beuther, H., Schilke, P., Menten, K. M., \& Wyrowski, F. 2002, ApJ, 566, 931

Tatematsu, K., Umemoto, T., Kameya, O., et al. 1993, ApJ, 404, 643

Terebey, S., Chandler, C. J., \& Andre, P. 1993, ApJ, 414, 759

Valdettaro, R., Palla, F., Brand, J., Cesaroni, R., Comoretto, G., \& Di, F. S. 2001, A\&A, 368, 845

Walmsley, C. M., \& Ungerechts, H. 1983, A\&A, 122, 164

Wang, Y., Wu, Y., Zhang, Q., Mao, R., \& Miller, M. 2005, A\&A, submitted

Wei, Y., Wu, Y., Wang, Y., Xu, D., \& Ju, B. 2005, JKAS, 38, 291

Wilking, B. A., Claussen, M. J., Benson, P. J., et al. 1994, ApJ, 431, L119

Wiseman, J. J., \& Ho, P. T. P. 1998, ApJ, 502, 676

Wood, D. O. S., \& Churchwell, Ed 1989a, ApJS, 69, 831

Wood, D. O. S., \& Churchwell, Ed 1989b, ApJ, 340, 265

Wouterloot, J. G. A., \& Brand, J. 1989, A\&AS, 80, 149

Wouterloot, J. G. A., Walmsley, C. M., \& Henkel, C. 1988, A\&A, 203, 367

Wu, Y., Wu, J., \& Wang, J. 2001, A\&A, 380, 665

Zhang, Q., Hunter, T. R., \& Sridharan, T. K. 1998, ApJ, 505, L151

Zhang, Q., Hunter, T. R., Brand, J., et al. 2001, ApJ, 552, L167 


\section{Online Material}


Y. Wu et al.: Ammonia cores in high mass star formation regions, Online Material p 2
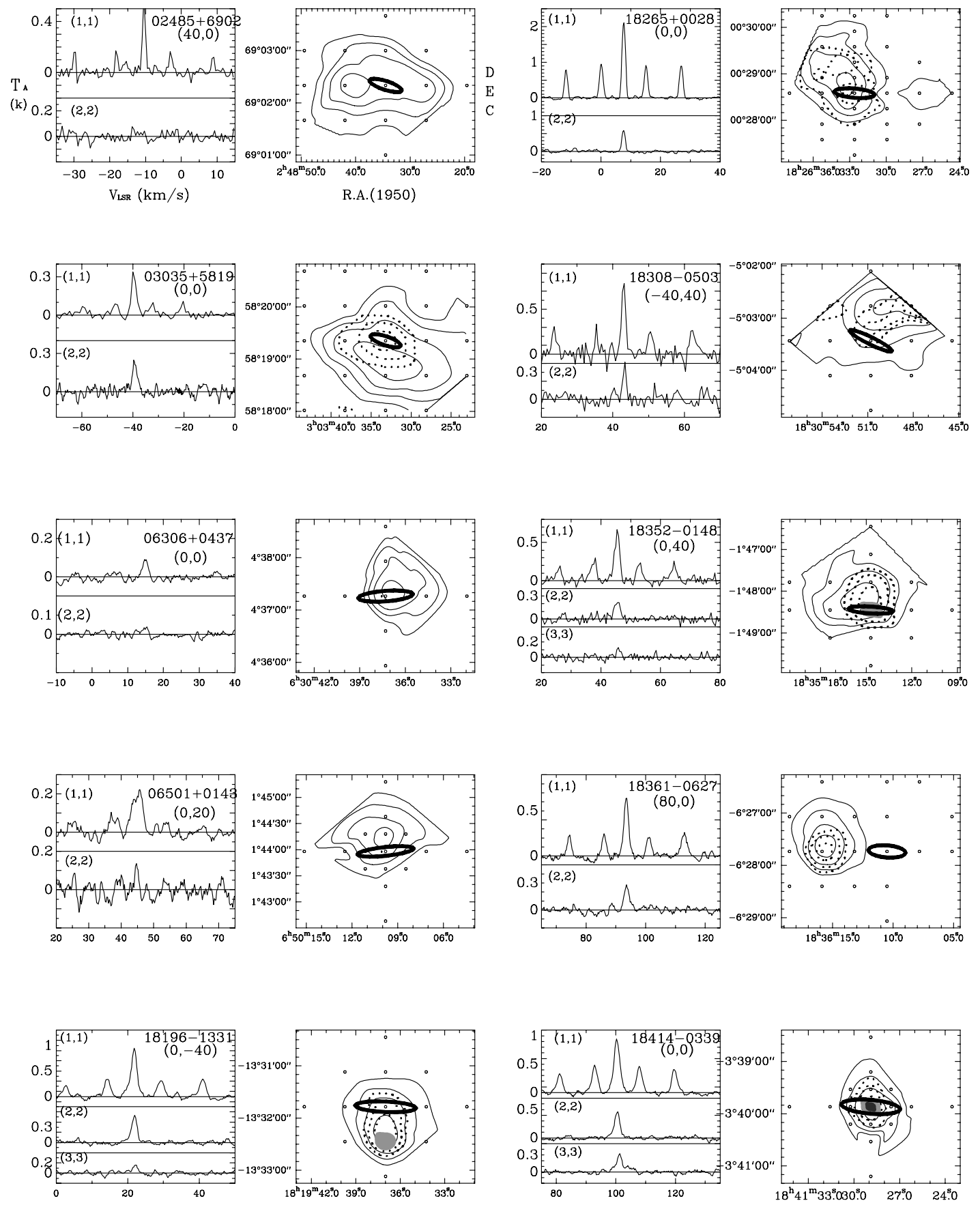

Fig. 1. $\mathrm{NH}_{3}$ spectra and velocity integrated intensity. For each source the left panel is the spectra and the right panel is the integrated emission. The solid and dashed contours show the $(1,1)$ and $(2,2)$ integrated intensity and the grey scales show the integrated intensity of the $(3,3)$ emission. The contours of the $(1,1)$ and $(2,2)$ intensity begin from $30 \%$ to $90 \%$ of the map peak intensity; those of $(3,3)$ begin from $65 \%$ with a step of $20 \%$. All the intensity peak values are listed in Table 2 as $P(1,1), P(2,2)$ and $P(3,3)$. The ellipse denotes the position and the uncertainty of the IRAS source. The source name and the position where the spectra were taken from are indicated at the upper-right corner of the spectral panel. 
Y. Wu et al.: Ammonia cores in high mass star formation regions, Online Material p 3
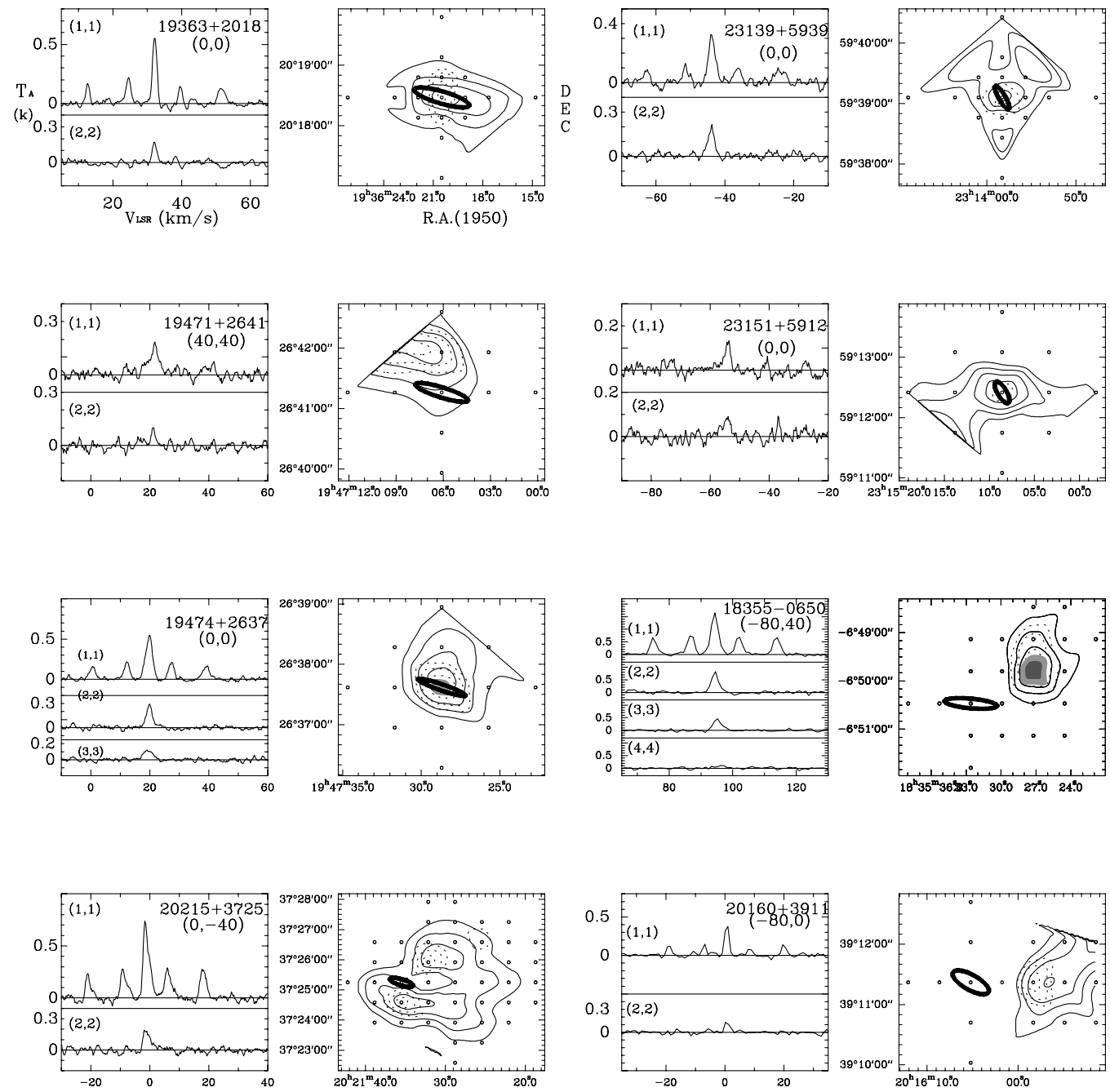

Fig. 1. continued. 
Y. Wu et al.: Ammonia cores in high mass star formation regions, Online Material p 4

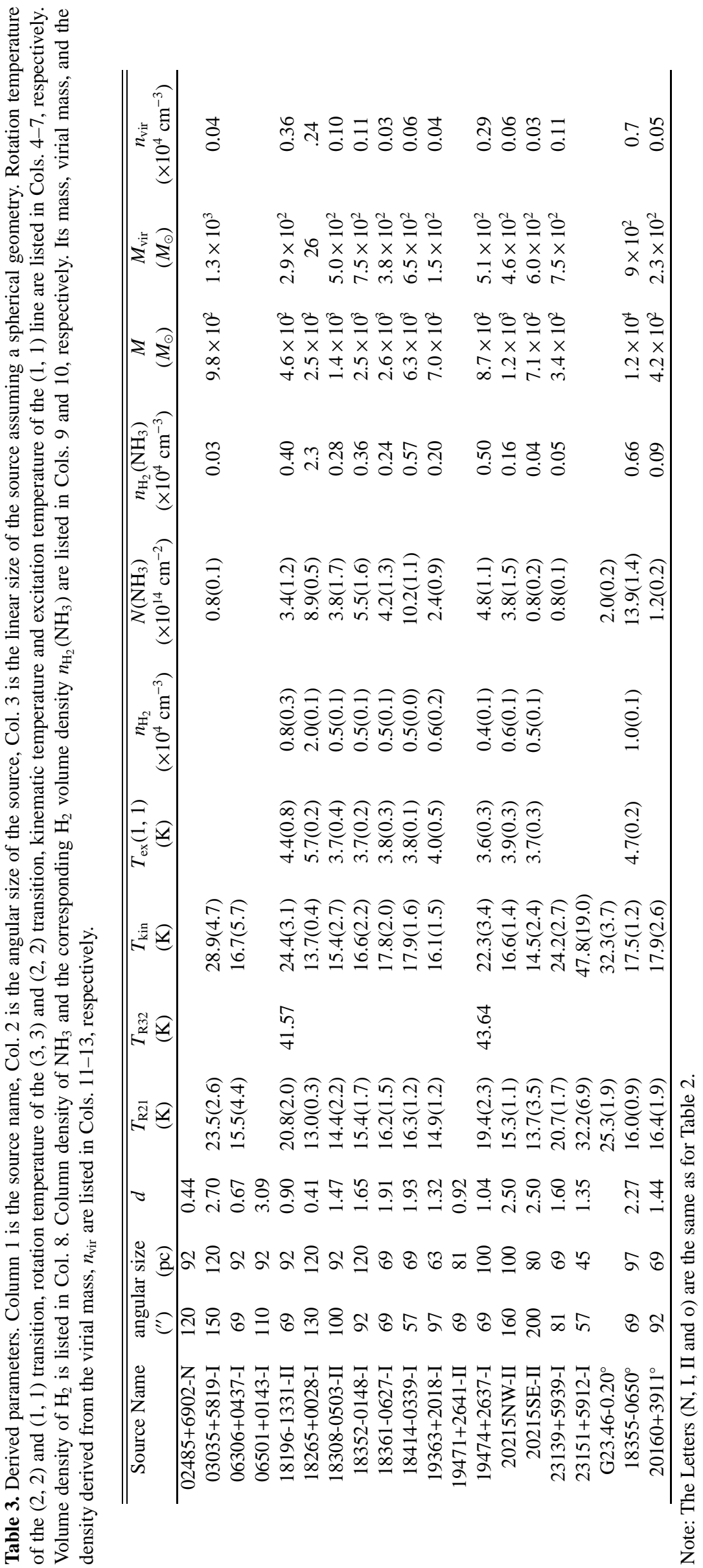




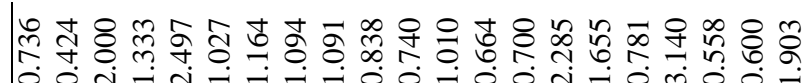
₹إ.

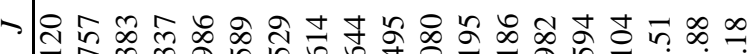

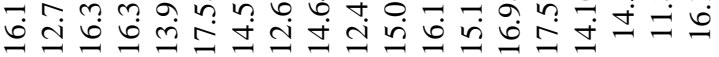

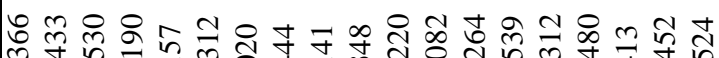

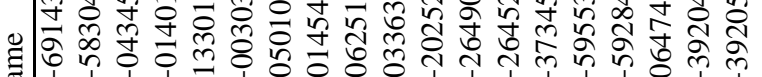

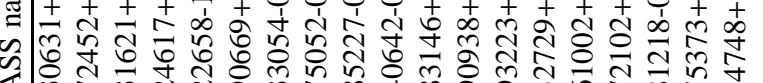

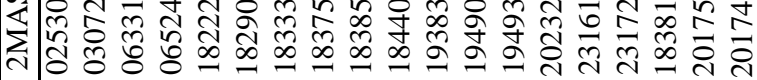

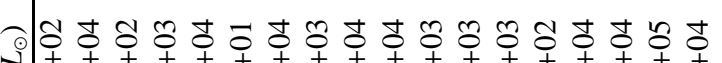

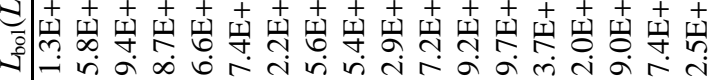

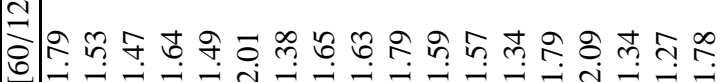
(b)

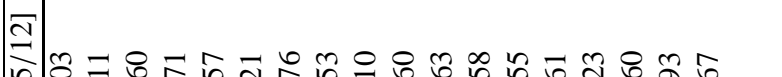
光 으

จ 可

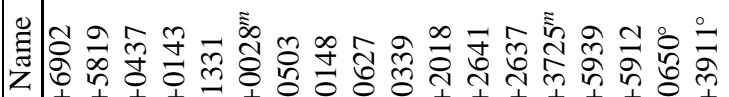

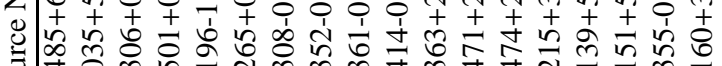

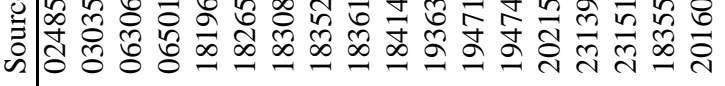

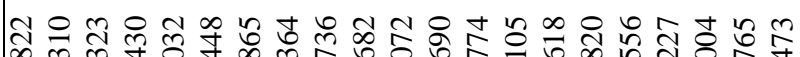

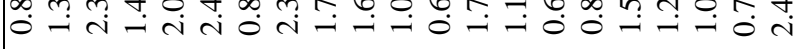

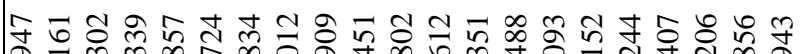

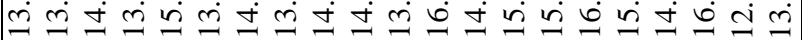

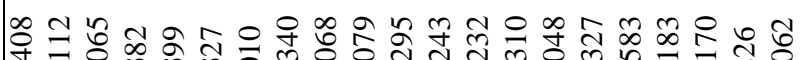

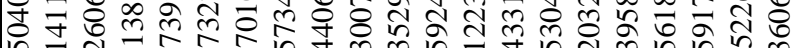

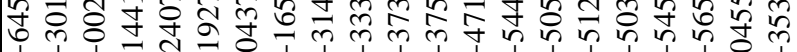

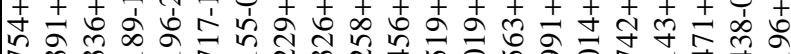
क人

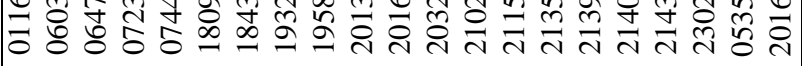
行

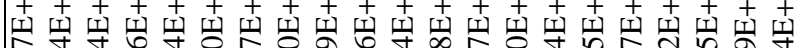

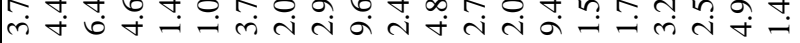

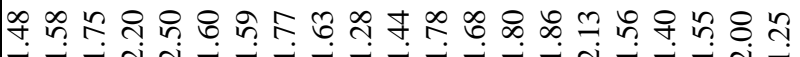

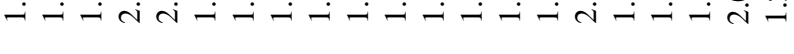

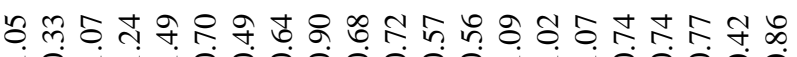
-i.

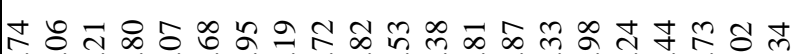

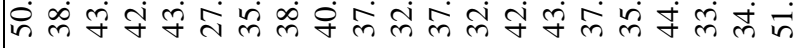

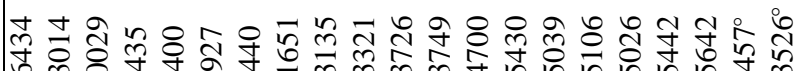

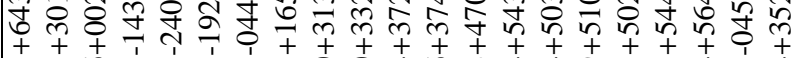

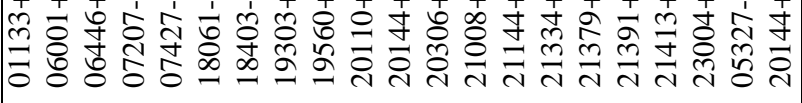

